\title{
A first-principle investigation of the Li diffusion mechanism in the super-ionic conductor lithium orthothioborate $\mathrm{Li}_{3} \mathrm{BS}_{3}$ structure.
}

\author{
F. Bianchini*, H. Fjellvåg, P. Vajeeston \\ Department of Chemistry, Centre for Materials Science and Nanotechnology, \\ University of Oslo, Norway, Box 1033 Blindern, N-0315 Oslo, Norway
}

\begin{abstract}
The research of superior ionic conductors is a very active field. Identifying such materials would allow for the design of improved solid-state Li-ion batteries, solving the safety hazard posed by the liquid electrolytes and improving the electrochemical stability and thus the energy efficiency. In this work, we study lithium orthothioborate $\mathrm{Li}_{3} \mathrm{BS}_{3}$ by means of fist-principle atomistic calculations based on Density Functional Theory. This material is a very promising super ion conductor candidate, as it is a layered compound based on a deformed body-centred structure of the anion sublattice. The minimum energy paths for the diffusion of the lithium ions are identified, and the activation energy are evaluated using the nudged elastic band method. A very fast $1 \mathrm{D}$ diffusive channel is found, contained in a Li-rich layer, with an activation energy below $0.1 \mathrm{eV}$. The other paths connecting these layers and extending the mobility of lithium to the whole structure are found to have activation barriers of $0.25 \mathrm{eV}$ or lower. This bottleneck corresponds to a diffusive coefficients of the order of $10^{-6} \mathrm{~cm}^{2} \mathrm{~s}^{-1}$, thus characterising lithium orthothioborate as an excellent ionic conductor.
\end{abstract}

Keywords: Li-ion battery, ionic conductivity, solid-state electrolyte, DFT

\footnotetext{
*Corresponding author

Email address: federico.bianchini@smn.uio.no (F. Bianchini)
} 


\section{Introduction}

Lithium-ion battery (LIB) is the most fast-growing battery technology, offering high energy density and superior mechanical properties. One of its key problems is the potential combustion hazard, caused by spewing liquid electrolyte vapours. The usage of solid-state electrolytes $(\mathrm{SE})$ is a viable solution to this issue, which would also ensure an improved efficiency due to a superior electrochemical stability. However, designing a solid-state electrolyte with ionic conductivity comparable to liquid ones is a demanding task. This challenge has been embraced by the scientific community, and many superior Li-ion conductors have been identified, including oxides such as as LISICON $\left(\mathrm{Li}_{14} \mathrm{ZnGe}_{4} \mathrm{O}_{16}\right)$ and garnet $\left(\mathrm{Li}_{7} \mathrm{La}_{3} \mathrm{Zr}_{2} \mathrm{O}_{12}\right)$ structures $[1,2]$ and oxynitride such as LIPON $\left(\mathrm{Li}_{2.8} \mathrm{PO}_{3.73} \mathrm{~N}_{0.14}\right)$ [3]. These compounds exhibit ionic conductivities in the range of $10^{-6}-10^{-3} \mathrm{~S} \mathrm{~cm}^{-1}$ at room temperature, with activation energies in the range $0.3-0.6 \mathrm{eV}$. More recently, a greater interest has been shown for sulphides, as they exhibit improved ion-diffusion properties, at the expense of a least good compatibility with the oxide cathodes. Some notable examples include the thio-LISICON $\mathrm{Li}_{3.25} \mathrm{Ge}_{0.25} \mathrm{P}_{0.75} \mathrm{~S}_{4}$ [4] and $\mathrm{Li}_{7} \mathrm{P}_{3} \mathrm{~S}_{11}$ [5], with activation energies in the $0.2-0.3 \mathrm{eV}$ range. In Ref. [6], the general criteria for superior ionic conductivity are investigated, finding a strong correlation between the body-centred cubic (bcc) anion sublattice and the low activation barriers. One of the proposed systems is lithium orthothioborate $\mathrm{Li}_{3} \mathrm{BS}_{3}$, a semi-planar material obtained by crystallisation of the corresponding glass [7] or by reaction of the metal sulphides, amorphous boron, and sulphur at $700^{\circ} \mathrm{C}$ [8]. This phase presents a planar trigonal boron coordination, not observed in the related $\mathrm{LiBS}_{2}$ and $\mathrm{Li}_{2} \mathrm{~B}_{4} \mathrm{~S}_{7}$ structures [9]. It presents excellent ionic mobility, as observed in Ref. [10] by far infrared and nuclear magnetic resonance spectroscopy. In this work, we investigate this compound by means of density functional theory (DFT). The $\mathrm{Li}_{3} \mathrm{BS}_{3}$ structure is optimised, and the minimum energy path (MEP) for the diffusion of lithium is identified, by calculating the relevant activation energies with the nudged elastic band (NEB) method.

\section{Computational Details}

DFT calculation are performed using the projected-augmented planewave (PAW) implementation of the Vienna ab initio simulation package (VASP) [11-14]. The Perdew, Burke, and Ernzerhof (PBE) functional [15] 
is adopted for the exchange-correlation term. Brillouin zone (BZ) integration is performed with $\Gamma$-centred Monkhorst-Pack grids [16], with a spacing of $0.15 \AA^{-1}$. A $500 \mathrm{eV}$ kinetic energy cutoff is used for the plane wave expansion, and the partial occupancies in reciprocal space are set according to the tetrahedron method with Blöchl corrections [17]. Our convergence tests, performed using a $0.15 \AA^{-1} \mathbf{k}$-points spacing and a $700 \mathrm{eV}$ cutoff energy, show that the energies are accurate within $5 \mathrm{meV}$ per formula unit. The optimised structure is obtained by minimising both the stress tensor and the Hellman-Feynman forces. The vacancy formation energy is evaluated as

$$
E_{v}^{f}=E_{\text {tot }}(N)-E_{\text {tot }}(N-1)-\mu
$$

where $E$ tot $(N)$ is the energy of a system with $N$ Li atoms, and $\mu$ is the chemical potential, set to the total energy of a fcc Li crystal. A $2 \times 1 \times 2$ supercell is used for calculation the formation and activation energies for the Li diffusion. The latter are evaluated with the climb-image Nudged Elastic Band (cNEB) method [18]. The transition path is modelled using five intermediate replicas, structurally optimised with a force convergence threshold of $20 \mathrm{meV} / \AA$. Ball-and-stick models are rendered with VESTA [19].

\section{Results and Discussion}

Lithium orthothioborate crystallises to an orthorhombic structure with space group Pnma (N. 62). Its equilibrium configuration, obtained from structural optimisation of the structure from Ref. [8], is displayed in Fig. 1(a). We report in Table 1 the equilibrium atomic positions and the lattice parameters. The latter are in excellent agreement with the experimental data. Two inequivalent Li atoms are observed, both exhibiting tetrahedral coordination. Atoms of the first type, labelled $\mathrm{Li}^{(1)}$ and indicated in light blue in Fig. 1, are in the same $(\boldsymbol{a}, \boldsymbol{c})$ layer, which we label ' $\mathrm{Li}^{(1)}$ layer'. $\mathrm{Li}^{(2)}$ atoms, positioned in the interlayer, are indicated in dark blue. The $\mathrm{Li}-\mathrm{S}$ bond length is calculated to be $2.48 \AA$. The boron atom, coplanar to $\mathrm{Li}^{(2)}$, presents a planar trigonal coordination group, with a B-S bond-length of $1.83 \AA$. The structure is therefore comprised by two kinds of $(\boldsymbol{a}, \boldsymbol{c})$ layers, one containing only atom of the $\mathrm{Li}^{1}$ type and the other containing one $\mathrm{Li}^{(2)}$ atom and the $\mathrm{BS}_{3}$ group. 
Table 1: Structural parameters for the optimised $\mathrm{Li}_{3} \mathrm{BS}_{3}$ structure and comparison with the experimental data. The fitting parameters to the equation of state are reported.

$$
\begin{aligned}
& a=8.1720 \AA b=10.1434 \AA c=6.1974 \AA \\
& a=8.1441 \AA b=10.0632 \AA c=6.1611 \AA \text { ref. [8] } \\
& V=128.687 \AA^{3} B=25.84 \mathrm{GPa} B^{\prime}=3.29 \\
& \text { atom Wyckoff } x \quad y \quad z \\
& \begin{array}{lllll}
\mathrm{B} & 4 \mathrm{c} & 0.12042 & 0.25000 & 0.88357
\end{array} \\
& \begin{array}{lllll}
\mathrm{Li}^{(1)} & 8 \mathrm{~d} & 0.66807 & 0.49060 & 0.39313
\end{array} \\
& \begin{array}{lllll}
\mathrm{Li}^{(2)} & 4 \mathrm{c} & 0.99504 & 0.25000 & 0.45405
\end{array} \\
& \begin{array}{lllll}
\mathrm{S}^{(1)} & 4 \mathrm{c} & 0.20213 & 0.25000 & 0.15916
\end{array} \\
& \begin{array}{lllll}
\mathrm{S}^{(2)} & 8 \mathrm{~d} & 0.91966 & 0.59424 & 0.25686
\end{array}
\end{aligned}
$$

Each $\mathrm{Li}^{(1)}$ atom has three neighbours in the layer, two at a $3.06 \AA$ distance and the other two at $3.38 \AA$, and two neighbours in the interlayer, at $3.55 \AA$ and $3.64 \AA . \mathrm{Li}^{(2)}$ atoms have four $\mathrm{Li}^{(2)}$ neighbours, two at $3.55 \AA$ and two $3.64 \AA$. The distance between $\mathrm{Li}^{(2)}$ atoms is $4.80 \AA$, larger than the previously described hops due to the presence of the coplanar $\mathrm{BS}_{3}$ group. This network is shown in Figure 1(b) with a ball-and-stick model, where sticks are connecting neighbouring $\mathrm{Li}$ atoms. The second $\mathrm{Li}^{(1)}-\mathrm{Li}^{(2)}$ pair and the $\mathrm{Li}^{(2)}-\mathrm{Li}^{(2)}$ one are not shown, as these hop are not involved in the diffusion of a Li-vacancy, as discussed later in this section.

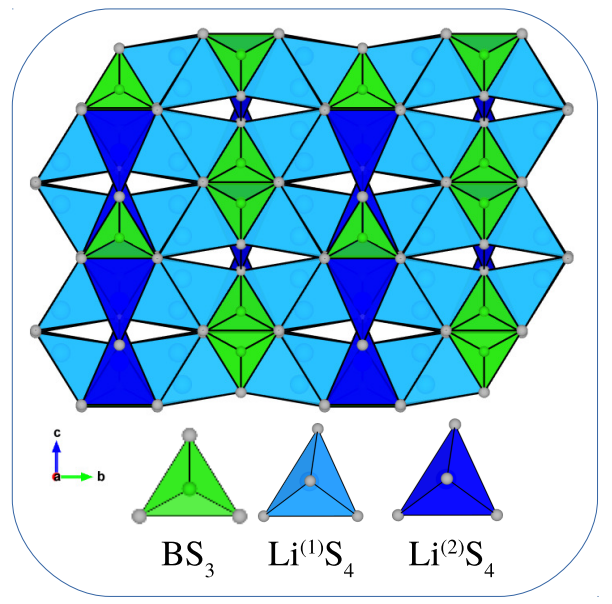

(a)

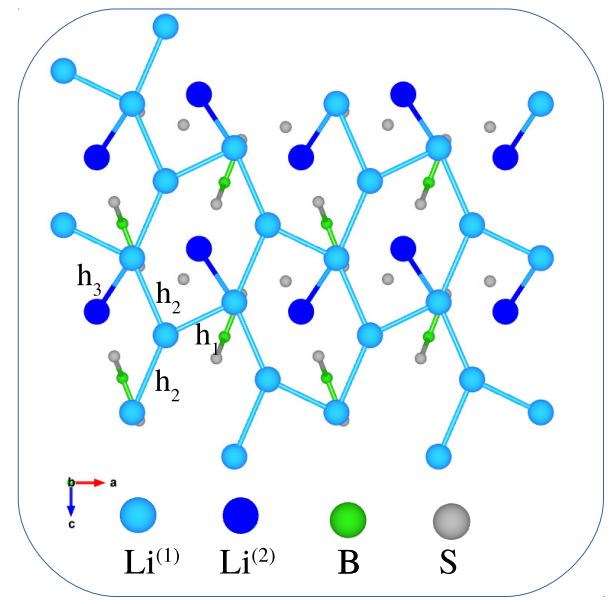

(b)

Figure 1: The equilibrium structure of $\mathrm{Li}_{3} \mathrm{BS}_{3}$ rendered with coordination polyhedra (a). Section of the $(a, c)$ layer (b). The hops $h_{n}$ to the nearest neighbours are indicated with sticks. These constitute the Li diffusive network. 
The vacancy formation energies for $\mathrm{Li}^{(1)}$ and $\mathrm{Li}^{(2)}$ are $3.31 \mathrm{eV}$ and $3.39 \mathrm{eV}$ respectively. This indicates that a vacancy is more likely to be found in the $\mathrm{Li}^{(1)}$ layer, but not unlikely to diffuse in the interlayer. This interlayer vacancy is then predicted to either return to the original position or to change $\mathrm{Li}^{(1)}$ layer, and not to diffuse in the interlayer, in virtue of the difference in formation energies. The activation barriers for the diffusion of a $\mathrm{Li}$ vacancy along the previously described nearest neighbours hops are calculated with the cNEB method. These hops are labelled $h_{n} n=1,2,3,4$, where $n$ is proportional to the distance from the vacancy. The diffusion along the $c$ direction involves only $h_{2}$ steps, with an activation barrier of $0.08 \mathrm{eV}$. Full diffusivity in the $(\boldsymbol{a}, \boldsymbol{c})$ layer requires also $h_{1}$ hops, found to have an activation barrier of $0.25 \mathrm{eV}$. This activation energy difference between $h_{1}$ and $h_{2}$ is explained in terms of the geometry of the MEP, shown in Figure 2(a) indicating the intermediate Li positions with small white spheres. Along the $h_{2}$ MEP, the Li atom moves out of the plane, forming a $\mathrm{LiBS}_{3}$ tetrahedron, which is a local minimum as appreciated from the energetics reported in Fig. 3. As the Li-B distance further decreases, the maximum energy along the MEP is reached. In the $h_{1}$ case the intermediate minimum is not observed, as the presence of two symmetrical $\mathrm{BS}_{3}$ groups hinders the deviation from the linear path.

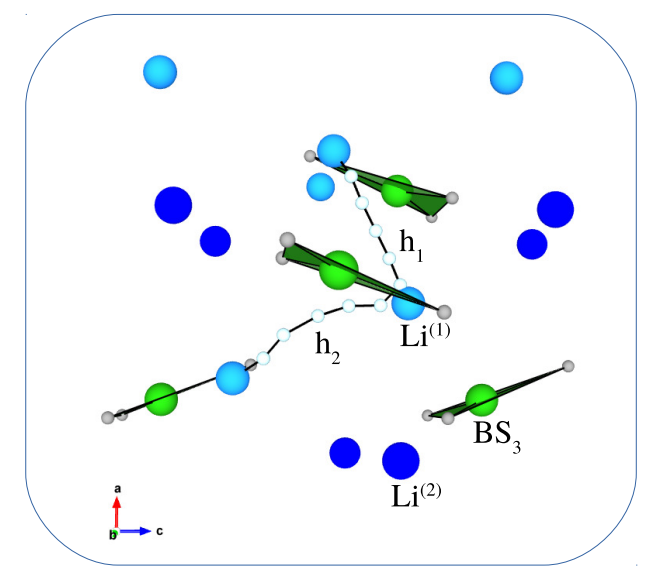

(a)

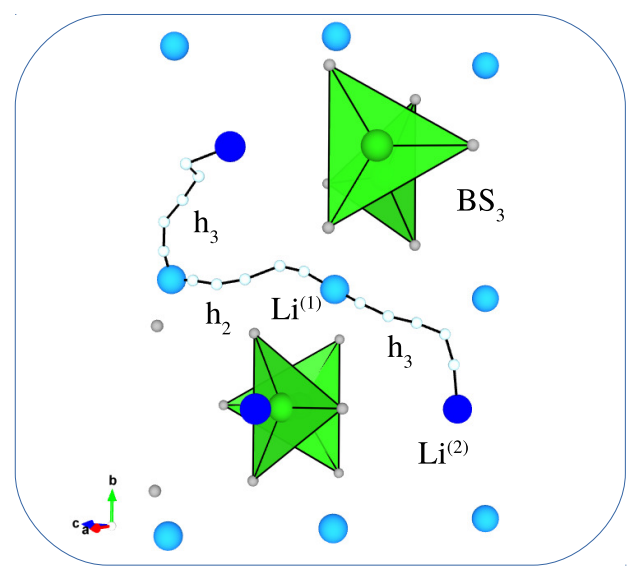

(b)

Figure 2: Positions of a Li vacancy along the MEP for diffusion in the $\mathrm{Li}^{(1)}$ layer (a) and along the orthogonal direction (b). 


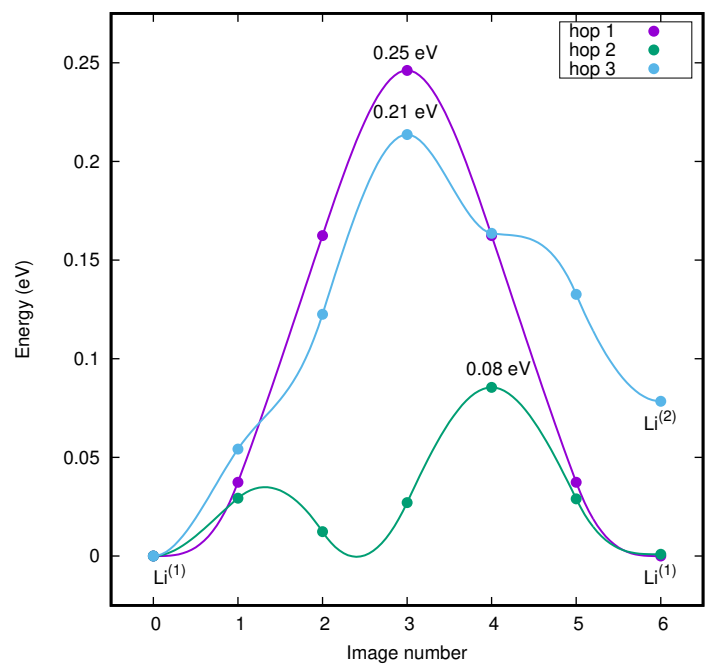

Figure 3: Energy profile along the MEP for the three vacancy hops required to achieve $3 \mathrm{D}$ diffusion in the $\mathrm{Li}_{3} \mathrm{BS}_{3}$ structure. The zero energy is fixed to the total energy of the system containing a vacancy in the $\mathrm{Li}^{(1)}$ site.

We now address the diffusion between $\mathrm{Li}^{(1)}$ layers. The $h_{3}$ and $h_{4}$ hops are found to have activation barriers of $0.21 \mathrm{eV}$ and $0.25 \mathrm{eV}$ respectively. We note that $h_{4}$ is unnecessary, as the diffusion along $\boldsymbol{b}$ can be achieved by a $h_{2}$ hop in the plane (activation energy $0.08 \mathrm{eV}$ ) followed by another $h_{3}$ hop. This is shown in Fig. 2(b) with ball-and-stick models, indicating the intermediate positions along the MEP. The energetics along the MEP is reported in Fig. 3. The bottleneck for diffusion along $\boldsymbol{b}$ is found to be the $h_{3}$ hop, with an activation energy of $0.21 \mathrm{eV}$, while in the plane the limiting step is $h_{1}$, with a $0.25 \mathrm{eV}$ barrier. Very high Li mobility is observed in the $1 \mathrm{D}$ channel along the $\boldsymbol{c}$ direction. Altogether, the $\mathrm{Li}_{3} \mathrm{BS}_{3}$ compound is shown to be an excellent Li ion conductor, with a full 3D mobility network, and a preferential diffusion channel along the $c$ direction.

\section{Conclusions}

We have provided a detailed description of the diffusion of $\mathrm{Li}$ ions in the $\mathrm{Li}_{3} \mathrm{BS}_{3}$ structure, identifying the minimum energy paths for vacancy hops. A fast 1D diffusion channel is observed in the Li-rich $(\boldsymbol{a}, \boldsymbol{c})$ layers along the $\boldsymbol{c}$ direction, connecting symmetrically equivalent Li sites. Diffusivity along $\boldsymbol{a}$ follows a two-hop mechanism. This MEP is contained the $(\boldsymbol{a}, \boldsymbol{c})$ layer, and the 
largest activation barrier is calculated to be $0.25 \mathrm{eV}$. The path connecting different $(\boldsymbol{a}, \boldsymbol{c})$ layers is also comprised by two steps, one of which in the $(\boldsymbol{a}, \boldsymbol{c})$ plane. The largest activation energy in this case is calculated for the hop between the inequivalent $\mathrm{Li}$ atoms, which allows for a vacancy to enter the interlayer with an activation energy of $0.21 \mathrm{eV} . \mathrm{Li}_{3} \mathrm{BS}_{3}$ is therefore found to have a 3D diffusion network, with a preferable diffusion channel along the $\mathbf{c}$ direction. None of the energy hops is found to have activation energy larger than $0.25 \mathrm{eV}$, corresponding to a diffusion coefficient of the order of $10^{-6} \mathrm{~cm}^{2} \mathrm{~s}^{-1}$. The system is thus shown to be an excellent ion-conductor, and a suitable solid electrolyte candidate.

\section{Acknowledgements}

The authors acknowledge the Research Council of Norway (Grant agreement SELiNaB-255441) for financial support and for providing the computer time (project number NN2875k) at the Norwegian supercomputer facility.

\section{Bibliography}

[1] P. Bruce, A. West, J. Electrochemical Soc. 130 (6) (1983) 662-669.

[2] R. Murugan, V. Thangadurai, W. Weppner, Angew. Chem. Int. Ed. 46 (41) (2007) 7778-7781.

[3] X. Yu, J. B. Bates, F. X. Hart, J. Electrochemical Soc. 144 (2) (1997) $524-532$.

[4] R. Kanno, M. Murayama, J. Electrochemical Soc. 148 (7) (2001) A742A746.

[5] H. Yamane, M. Shibata, Y. Shimane, T. Junke, Y. Seino, S. Adams, K. Minami, A. Hayashi, M. Tatsumisago, Solid State Ion. 178 (15) (2007) $1163-1167$.

[6] Y. Wang, W. D. Richards, S. P. Ong, L. J. Miara, J. C. Kim, Y. Mo, G. Ceder, Nat. Mater. 14 (10) (2015) 1026-1031.

[7] P. Vinatier, P. Gravereau, M. Ménétrier, L. Trut, A. Levasseur, Acta Crystallogr. C 50 (8) (1994) 1180-1183. 
[8] C. J. F. Hiltmann, B. Krebs, Z. anorg. allg. Chem. 622 (1996) 1508-1514.

[9] J. Cho, S. W. Martin, J. Non-Cryst. Solids 170 (2) (1994) 182 - 189.

[10] P. Vinatier, M. Ménétrier, A. Levasseur, Phys. Chem. Glasses 44 (2) (2003) 135-142.

[11] G. Kresse, J. Hafner, Phys. Rev. B 47 (1993) 558-561.

[12] G. Kresse, J. Furthmüller, Comput. Mater. Sci. 6 (1) (1996) 15-50.

[13] G. Kresse, J. Furthmüller, Phys. Rev. B 54 (1996) 11169-11186.

[14] G. Kresse, D. Joubert, Phys. Rev. B 59 (1999) 1758-1775.

[15] J. P. Perdew, K. Burke, M. Ernzerhof, Phys. Rev. Lett. 77 (1996) 38653868.

[16] H. J. Monkhorst, J. D. Pack, Phys. Rev. B 13 (12) (1976) 5188-5192.

[17] P. E. Blöchl, O. Jepsen, O. K. Andersen, Phys. Rev. B 49 (1994) 1622316233.

[18] G. Henkelman, B. P. Uberuaga, H. Jónsson, J. Chem. Phys. 113 (22) (2000) 9901-9904.

[19] K. Momma, F. Izumi, J. Appl. Crystallogr. 41 (3) (2008) 653-658. 